\title{
Study of the influence of a grid on the plasma in a single-ended Q-machine
}

\author{
Andersen, S.A.; Jensen, Vagn Orla; Nielsen, P.
}

Published in:

Physics of Fluids

Link to article, DOI:

$10.1063 / 1.1692412$

Publication date:

1969

Document Version

Publisher's PDF, also known as Version of record

Link back to DTU Orbit

Citation (APA):

Andersen, S. A., Jensen, V. O., \& Nielsen, P. (1969). Study of the influence of a grid on the plasma in a singleended Q-machine. Physics of Fluids, 12, 2694-2701. https://doi.org/10.1063/1.1692412

\section{General rights}

Copyright and moral rights for the publications made accessible in the public portal are retained by the authors and/or other copyright owners and it is a condition of accessing publications that users recognise and abide by the legal requirements associated with these rights.

- Users may download and print one copy of any publication from the public portal for the purpose of private study or research.

- You may not further distribute the material or use it for any profit-making activity or commercial gain

- You may freely distribute the URL identifying the publication in the public portal

If you believe that this document breaches copyright please contact us providing details, and we will remove access to the work immediately and investigate your claim 


\title{
Study of the Influence of a Grid on the Plasma in a Single-Ended $Q$-Machine
}

\author{
S. A. Andersen, V. O. Jensen, and P. Nielsen \\ Atomic Energy Commission, Research Establishment Risö, Roskilde, Denmark
}

(Received 24 March 1969)

\begin{abstract}
The mechanism of the interaction between a biased grid and the plasma in a "single-ended" $Q-$ machine was studied experimentally. It was found that the grid reflects some of the ions in the plasma flow. The number and velocity distribution of the reflected ions depend on the bias of the grid, on the plasma density, and on the distance between the grid wires. The reflected ions move as freely streaming particles except at high densities, where binary collisions are of importance. A small sinusoidal voltage applied to the biased grid excites an upstream wave with dispersion properties that can be explained as phase mixing of freely streaming particles reflected by the grid.
\end{abstract}

\section{INTRODUCTION}

Ion-acoustic waves in cesium and potassium plasmas in $Q$-machines have been studied intensively in recent years. Wong, D'Angelo, and Motley ${ }^{1}$ reported on experimental results obtained in collisionless plasmas which were in accordance with the theoretically predicted Landau damping. Later, Motley and Wong ${ }^{2}$ showed that in high-density plasmas the damping of ion-acoustic waves can be explained as viscous damping. The influence of ion-neutral collisions has been studied by Andersen et $a l .{ }^{3}$ and by Doucet and Gresillon. ${ }^{4}$ Recently, measurements of nonlinear effects have been reported by Buzzi, Gresillon, and Doucet $^{5}$ and by Ikezi and Takahashi. ${ }^{6}$ Finally, work on ion wave echoes has been performed by Baker et $a l^{7}$ and by Ikezi and Takahashi. ${ }^{8}$

In all the experiments mentioned above the waves were generated by a grid placed in the plasma column with its surface perpendicular to the magnetic lines of force. A sinusoidal voltage oscillating around a negative bias on the grid excited the waves. To the best of our knowledge nobody has performed a

1 A. Y. Wong, N. D'Angelo, and R. W. Motley, Phys. Rev. Letters 9, 415 (1962); Phys. Rev. 133, A436 (1964).

9 R. W. Motley and A. Y. Wong, in Proceedings of the Sixth International Conference on Ionization Phenomena in Gases, P. Hubert and E. Crémien, Eds. (Bureau des Editions, Centre d'Etudes Nucléaires de Saclay, Paris, 1963), Vol. 3, p. 133.

H. K. Andersen, N. D'Angelo, V. O. Jensen, P. Michelsen, and $P$. Nielsen, Phys. Fluids 11, 1177 (1968).

${ }^{4}$ H. J. Doucet and D. Gresillon, in Plasma Physics and Controlled Nuclear Fusion Research (International Atomic Energy Agency, Vienna, 1969), Vol. I, p. 659.

J. M. Buzzi, D. Gresillon, and H. J. Doucet, Bull. Am. Phys. Soc. 13, 1569 (1968).

- N. Sato, H. Ikezi, N. Takahashi, Y. Yamashita, and T Obiki, Institute of Plasma Physics, Nagoya University, Japan, Report IPPJ-62 (1967).

T D. R. Baker, N. R. Ahern, and A. Y. Wong, Phys. Rev. Letters 20,318 (1968).

${ }^{8}$ H. Ikezi, N. Takahashi, and K. Nishikawa, Phys. Fluids $12,853(1969)$. comprehensive study of the mechanism by which the grid excites the waves.

It is normally assumed that the grid changes the ion density by changing the number of ions collected. The density perturbation propagates as an ion-acoustic wave. The dispersion properties of this wave have been determined with another negatively biased grid (or Langmuir probe), which measures the density perturbation as a function of the distance from the exciting grid and of time. The aim of most of the experiments has been to verify Landau's ${ }^{9}$ theory for waves in collisionless plasmas or other theoretical calculations based on the same model. ${ }^{10,11}$ When experimental results have been compared with theories, it has been assumed that details in the exciting mechanism have no or little influence on the propagation of the waves. In the theories this corresponds to disregarding the effects of the initial perturbation in the distribution function and only considering the so-called natural modes in the plasma. As discussed by Hirshfield and $\mathrm{Jacob}^{12}$, disregard of the unknown initial perturbation seems to be poorly justified, especially for measurements taken close to the exciting grid, where the perturbation has not disappeared by phase mixing.

In this paper we describe measurements made in order to examine the mechanism by which a biased grid influences a $Q$-machine plasma. We have been especially interested in the perturbation in the velocity distribution around a grid with changing potential. The experiments were made in the Risö $Q$-machine, which was operated "single ended" with only one hot plate. Different grids were placed in

\footnotetext{
L. Landau, J. Phys. (USSR) 10, 25 (1946).

${ }^{10}$ R. W. Gould, Phys. Rev. 136, A991 (1964).

11 R. W. Gould, T. M. O'Neil, and J. H. Malmberg, Phys. Rev. Letters 19, 219 (1967).

12 J. L. Hirshfield and J. H. Jacob, Phys. Fluids 11, 411 (1968).
} 
the column, and the changes in the plasma properties between the hot plate and the grid were studied as a function of grid voltage.

The essential results of our experiments are as follows:

(a) A negatively biased grid reflects some of the ions in the plasma flow. The number and the velocity distribution of reflected ions depend on the grid voltage. Generally, a more negative grid voltage corresponds to reflection of particles with higher velocities.

(b) In not-too-dense plasmas the reflected ions are found to move as freely streaming particles. At higher densities $\left(n \gtrsim 10^{11} \mathrm{~cm}^{-3}\right)$ the interaction between the reflected particles and the main plasma flow can be explained by binary collisions.

(c) When a sinusoidal voltage is applied to a negatively biased grid, a wave with a phase velocity away from the grid is excited. The dispersion of this wave can be explained in a model with freely streaming particles, i.e., the damping can be explained as caused by phase mixing rather than Landau damping.

Section II of this paper contains a discription of the experimental set-up. In Sec. III we describe the experiments made in order to explain the interaction between grid and plasma, and propose a physical picture for this interaction. In the last section, we examine the interaction between the reflected particles and the main plasma.

\section{EXPERIMENTAL SET-UP}

A single-ended $Q$-device as shown schematically in Fig. 1 was used for this experiment. Only one Ta plate was heated, the cold one was either grounded or left electrically floating. Thus, we had a continuous plasma flow from the hot to the cold plate. The density of the flow could be changed by changing the oven temperature. Different grids were placed in the flow, and the change in the upstream plasma as a function of grid voltage was studied by means of a shielded twin probe, ${ }^{13}$ consisting of two $0.2-\mathrm{mm}$ diam stainless-steel wires, $4 \mathrm{~cm}$ long. The shield consisted of two stainless-steel tubes, $1.5 \mathrm{~mm}$ diam, cut in half along their axes and welded together. In Fig. 1 a cross section of the twin probe can be seen. In use, the shield was left electrically floating. When oriented as in Fig. 1 and when biased to collect ions $\left(V_{\text {probe }} \approx-20 \mathrm{~V}\right)$, the front probe is assumed

13 S. A. Anderson, V. O. Jensen, P. Nielsen, and N. D'Angelo, Phys. Fluids 12, 557 (1969).
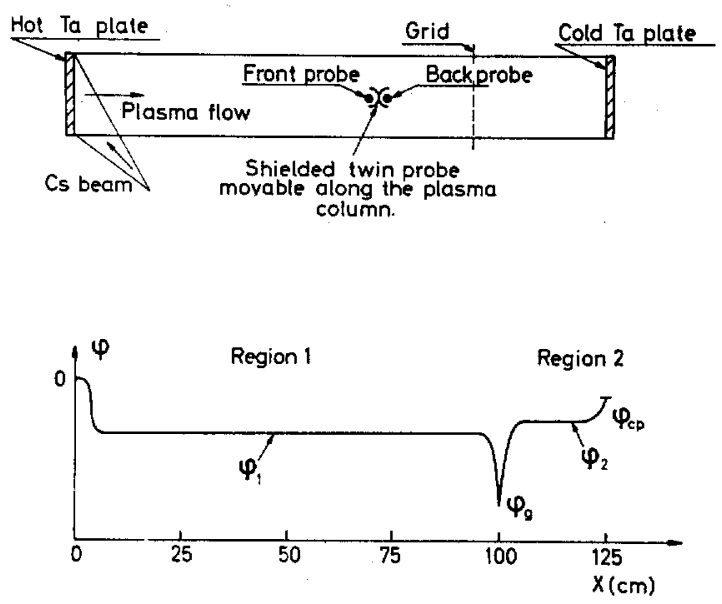

Fig. 1. Experimental set-up and schematic of the plasma potential.

to measure a signal proportional to

$$
\int_{0}^{\infty} v_{\|} f\left(v_{\|}\right) d v_{n}
$$

where $f\left(v_{i}\right)$ is the distribution over velocities parallel to the magnetic field. Similarly, the back probe measures a signal proportional to

$$
\int_{-\infty}^{0} v_{1} f\left(v_{11}\right) d v_{1} .
$$

All the experiments reported in this paper were performed in Cs plasmas. The strength of the magnetic field was always around $8 \mathrm{kG}$, corresponding to an ion Larmor radius of around $1 \mathrm{~mm}$.

\section{INTERACTION BETWEEN GRID AND PLASMA}

\section{A. Direct Current Measurements}

When the grid is left electrically floating, the front probe measures a signal 10 to 100 times larger than that measured by the back probe. This shows that the plasma is drifting from the hot to the cold plate. ${ }^{13}$ No change in the two signals is seen if the grid is drawn out of the plasma. When the grid voltage is decreased below floating potential, the change in the front probe signal is very small, while we get an increase in the back probe signal depending on the grid voltage. Figure 2(a) shows typical characteristics of the two probes as functions of the dc-voltage on the grid. Both probes were biased $20 \mathrm{~V}$ negatively, and the ion current was measured by the voltage drop across a 1-k $\Omega$ resistor. Characteristics like the one shown in Fig. 2(a) were taken for different plasma densities (measured by the ion saturation current to the front probe) and with different grids. Three grids were used, all made of $0.02-\mathrm{mm}$-diam 
(a)

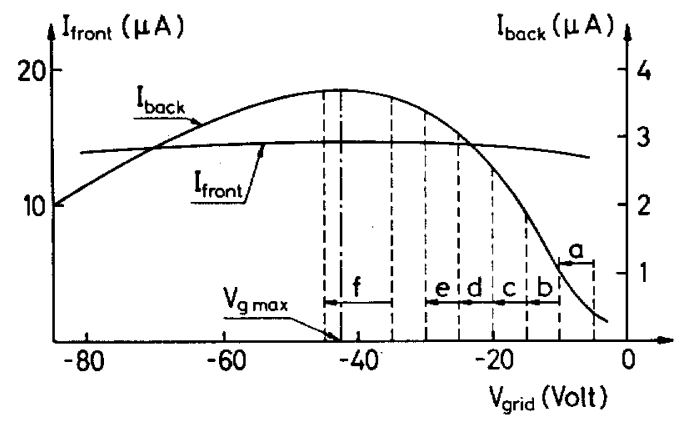

(b)

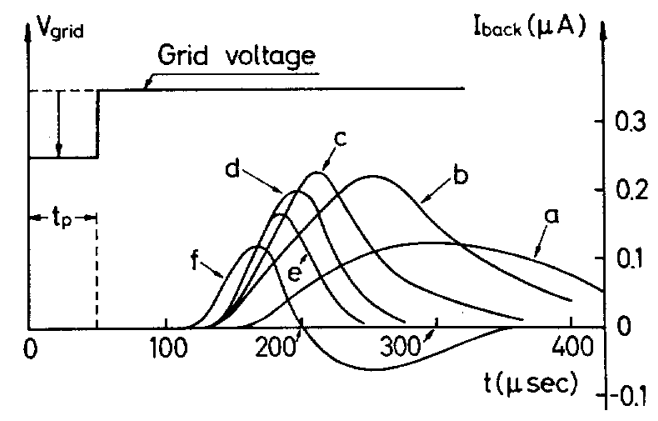

FIG. 2. (a) Direct current characteristics, $n \approx 1.5 \times 10^{10}$ $\mathrm{cm}^{-3}$, grid spacing $2 \mathrm{~mm}$. (b) Redrawings of reflected pulses. Distance between grid and twin probe $=30 \mathrm{~cm}$.

$\mathrm{Cu}$ wire wound on a stainless-steel frame, the spacing between the wires being $0.3,1$, and $2 \mathrm{~mm}$, respectively. For all densities in the region $10^{9}<n<$ $10^{11} \mathrm{~cm}^{-3}$ and for all three grids the characteristics show the same main features as the one in Fig. 2(a): at floating grid potential no reflection takes place; when the potential $V_{g}$ is decreased, the back current $I_{\text {back }}$ increases and reaches a maximum at $V_{o \text { max }}$. For further decreasing grid voltages $I_{\text {back }}$ decreases and asymptotically approaches zero for very negative values of $V_{g}$. For increasing densities or increased spacing between grid wires a more negative voltage is required for maximum reflection to be reached. Table I shows the measured quantities $V_{g \max }$ and $\left(I_{\text {back }} / I_{\text {front }}\right)_{\max }$ for different densities and grids.

\section{B. Pulsed Grid}

In order to find the velocity distribution of the reflected particles we pulsed the voltage on the grid and measured the time dependence of the signal received on the back probe. Assuming that the reflected ions move as freely streaming particles, the time dependence of the signal gives the velocity distribution. This is a simple time-of-flight measurement. Evidence that the ions do move as free particles will be given in Sec. IV. For an accurate determination of the velocity distribution the grid pulse
Table I. Variation of $V_{\text {gmax }}$ and $I_{\text {back }} / I_{\text {f roat }}$ with density and wire spacing.

\begin{tabular}{ccccc}
\hline \hline \multirow{2}{*}{$\begin{array}{c}\text { Density } \\
\left(\mathrm{cm}^{-3}\right)\end{array}$} & & $0.3 \mathrm{~mm}$ & $1 \mathrm{~mm}$ & $2 \mathrm{~mm}$ \\
\cline { 3 - 5 } & & & \multicolumn{3}{c}{ Grid spacing } \\
\hline $7 \times 10^{9}$ & $V_{g \max }(\mathrm{V})$ & -5 & -26 & -24 \\
& $\left(I_{b} / I_{f}\right)_{\max }$ & 0.5 & 0.3 & 0.3 \\
$1.6 \times 10^{10}$ & $V_{b \max }(\mathrm{V})$ & -10 & -18 & -40 \\
& $\left(I_{b} / I_{f}\right)_{\max }$ & 0.4 & 0.3 & 0.3 \\
$3 \times 10^{10}$ & $V_{g \max }(\mathrm{V})$ & -14 & -26 & -60 \\
& $\left(I_{b} / I_{f}\right)_{\max }$ & 0.4 & 0.3 & 0.3 \\
\hline
\end{tabular}

was kept short as compared with the pulse received on the probe. Figure 2(b) shows redrawings of typical oscilloscope pictures. The upper beam shows the grid voltage. From a de value a negative square pulse of duration $50 \mu \mathrm{sec}$ is applied to the grid as shown to the left in the picture. The redrawings of the lower beam show a family of curves, all corresponding to the ac signal received on the back probe. For the curve a, the dc grid voltage was $-5 \mathrm{~V}$, and the amplitude of the pulse was $-5 \mathrm{~V}$; for $b$ the de voltage was $-10 \mathrm{~V}$ and the amplitude again $-5 \mathrm{~V}$. Comparison with Fig. 2(a) will give the corresponding values for the remaining curves in the family.

From pictures like the ones in Figs. 2(b) and 3(b) the distribution over velocities parallel to the magnetic field has been deduced. It is found that drifting Maxwellian distributions fit the measured curves within the uncertainty. As is clearly seen from Figs. 2(b) and 3(b), the drift velocity of the reflected ions increases with negative grid pulse. For small pulses it is around the ion thermal velocity, increasing to about twice this velocity for grid pulses going down to about the maximum of the characteristic in Fig. 2(a). For pulses more negative than corresponding to the maximum, the situation is more complicated [see curve $f$ in Fig. 2(b)]. The temperature of the reflected ions is very close to that of the main plasma. $(T \simeq 0.2 \mathrm{eV})$.

\section{Plate Behind Grid}

In order to investigate the reflection mechanism in more detail we placed a metal plate $1 \mathrm{~mm}$ behind the grid (on the downstream side). For the experiments described in this subsection a crosswound grid, mesh $1.0 \mathrm{~mm}$, was used.

In the first experiment the pulse of reflected ions on the back probe was measured for different de voltages on the plate. Figure $3(\mathrm{a})$ shows reflected pulses for six different plate voltages $V_{p}$ between 
floating and $-28 \mathrm{~V}$; the grid was pulsed from a dc voltage of -5 to $-17 \mathrm{~V}$ (pulse length, $t_{p}=50 \mu \mathrm{sec}$ ). The figure shows that the amplitude of the reflected pulse decreases with decreasing $V_{p}$ while its shape is only slightly changed. Figure $3(\mathrm{~b})$ presents the corresponding pulse for a case where the plate was floating while the grid was pulsed from floating to voltages from -15 to $-2.5 \mathrm{~V}$. Here, we see that the number as well as the velocity of the reflected particles decrease with the amplitude of the grid pulse.

In the second experiment mentioned in this subsection, grid current-voltage characteristics were measured at different plate voltages. Figure 4(a) shows a series of oscilloscope pictures. When the plate is floating, the characteristic has the normal shape: ion current monotonely increasing with negative voltage. When the plate voltage is lowered, the characteristic changes gradually. First, we get a decrease in the ion current for low grid voltages. Eventually, this decrease is so large that we obtain a maximum on the curves. For even lower plate voltages this maximum moves to the right and becomes less and less pronounced. At very low $V_{p}$, it disappears, and we are left with a nearly constant ion current, which is much smaller than the one found with the plate floating. The plate voltage at which the maximum of the characteristic just disappears is called $V_{p}^{\prime}$. It is plotted as a function of density in Fig. 4(b).

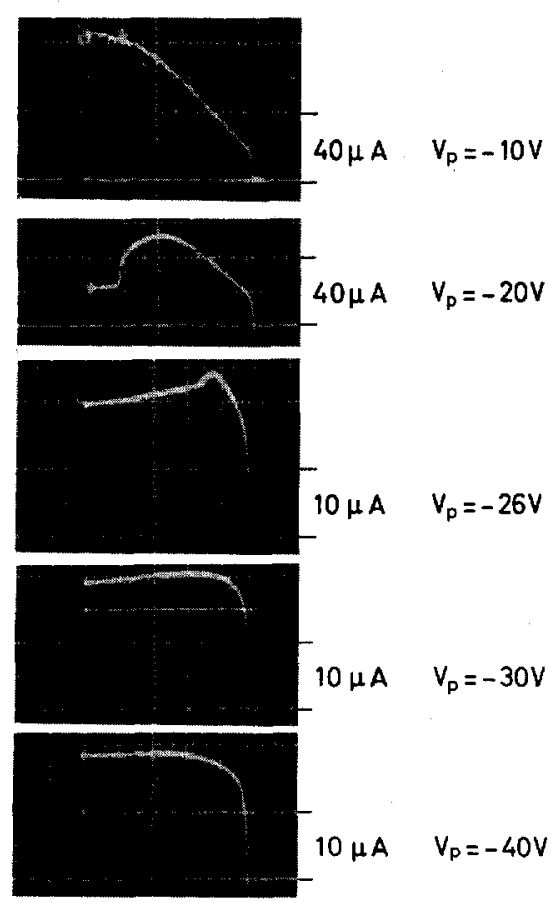

(a)

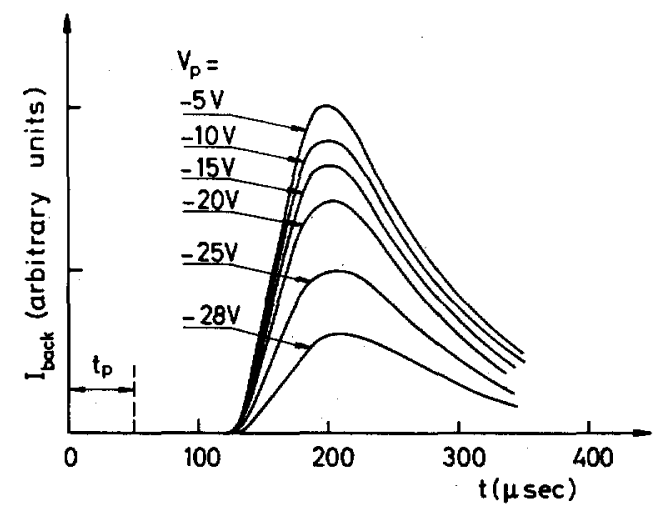

(a)

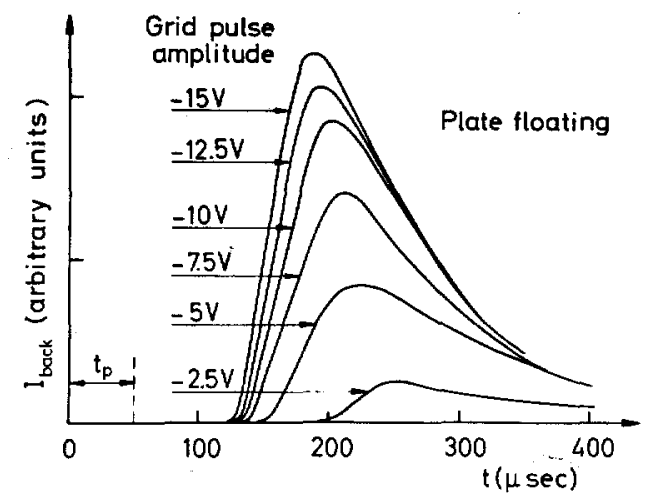

FrG. 3. Redrawings of reflected pulses with plate behind grid. Distance between plate and grid $=1 \mathrm{~mm}$. Distance between grid and twin probe $=30 \mathrm{~cm}$. (a) Plate voltage varied between -5 and $-28 \mathrm{~V}$. Grid pulsed from $-5 \mathrm{~V}$ dc to -17 V. (b) Plate floating. Grid pulse amplitude varied between -2.5 and $-15 \mathrm{~V}$. Grid de voltage $-5 \mathrm{~V}$.

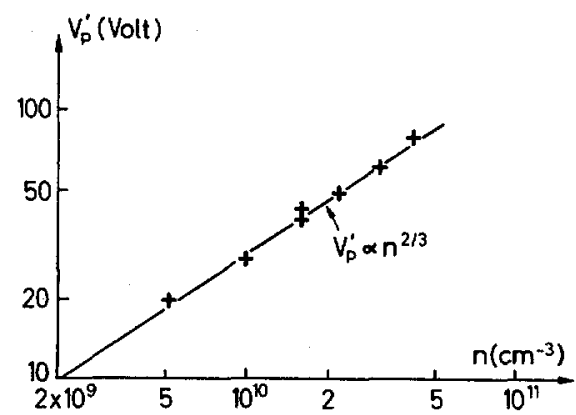

(b)

FIG. 4. (a) Family of grid characteristics for different voltages on plate behind grid. Sweep $20 \mathrm{~V} / \mathrm{cm} . \quad n=1.5 \times$ $10^{10} \mathrm{~cm}^{-3} \cdot V_{p}^{\prime}=-40 \mathrm{~V}$ determined from this family. (b) $V_{p}^{\prime}$ as a function of $n$ determined from families like the one shown in (a). 


\section{Echo Measurements}

As the final part of the investigation it was examined whether an echo could be reflected by a grid. Two grids were immersed in the plasma column. The distance between them was $L=23 \mathrm{~cm}$. The voltage on the first grid $g_{1}$ (closest to the hot plate), was oscillated around a negative bias with a frequency $\omega_{1}=15 \mathrm{kc} / \mathrm{sec}$. Similarly, the voltage on the other grid $g_{2}$ was oscillated with a frequency $\omega_{2}=50 \mathrm{kc} / \mathrm{sec}$ around a negative bias. According to theory ${ }^{11}$ and experiments ${ }^{7,8}$ an echo with the frequency $\omega_{2}-\omega_{1}=35 \mathrm{kc} / \mathrm{sec}$ should occur at a distance $l=L \omega_{1} /\left(\omega_{2}-\omega_{1}\right) \simeq 10 \mathrm{~cm}$ on the downstream side of $g_{2}$. This echo was seen on the front side of the twin probe when it was moved to that position. The phase velocity of the echo was found to be in the flow direction. Similarly, the back probe detected an echo with the same frequency at a distance $l$ from $g_{2}$ in the upstream direction (between the grids). The phase velocity of this echo was against the flow. The amplitudes of the echoes were measured relative to the dc signals of the probes. The relative amplitude of the reflected echo was characteristically $\frac{1}{3}$ of that of the transmitted one. Our observation of a reflected echo contradicts the experiment of Ref. 8 , where such an echo could not be detected.

\section{E. Discussion of the Reflection Mechanism}

The experiments described in the preceding subsections suggest the physical picture of the interaction between grid and plasma, which is discussed in the following.

We first examine the effects of an idealized grid consisting of an infinite number of infinitely thin wires that do not absorb ions. In this case, the problem is one-dimensional, and the potential along the plasma column looks like the sketch in Fig. 1. In the single-ended arrangement electrons are emitted from the hot plate at $x=0$ only. When $\varphi_{g}$ is negative, the potential wall for electrons in front of the grid reflects some of the electrons, the number increasing with the negative grid potential. If no potential difference between the two regions exists $\left(\varphi_{1}=\varphi_{2}\right)$, all ions can pass the grid. As a negative change in $\varphi_{g}$ decreases the electron flow into region 2 , the ion flow also has to decrease. This flow can only decrease if a potential jump is set up in the plasma $\left(\varphi_{2}>\varphi_{1}\right)$. Therefore, a negatively biased grid in a single-ended $Q$-machine will create a potential jump in the plasma which increases with decreasing grid potential.

The qualitative picture of the changes in the potential jump with $\varphi_{a}$ can explain some of the observations described above. When the grid is floating, it has no influence on the potentials, and therefore, $\varphi_{1}=\varphi_{2}$. As soon as a negative voltage is applied to the grid, a potential barrier for the drifting ions is built up around the grid. When this barrier is small (small $V_{g}$ ), only ions with a low parallel velocity will be reflected while fast particles can penetrate. As $V_{g}$ is decreased, the barrier increases, and faster ions are reflected. This agrees with the curves in Figs. 2 and 3(b) which show that the number of ions reflected from the grid and their averaged velocity increase with $V_{g}$.

By changing the bias on a plate close behind the grid one would expect to be able to change the potential barrier that reflects the ions. Figure 3(a). shows that when $V_{p}$ is made more negative, fewer ions are reflected. Also, a change in the distribution function toward lower velocities would be expected when $V_{p}$ is made more negative. Only a very slight change in the velocity is seen in Fig. 3(a). This shows that a lowering of the potential barrier is not a sufficient explanation.

In order to explain why the characteristics described in Sec. IIIA each have a maximum, one has to include the ions lost on the grid. In the discussion just given these were neglected and the potential well was assumed to have a plane shape across the column without irregularities. This is not the case because the grid is made up of a finite number of wires. The potential well is deeper close to a wire than midway between two wires. At small negative grid voltages only a few ions will fall into the deep potential wells, and the discussion given above is valid. As the grid is made more negative, the wells around the wires penetrate farther into the plasma, and more ions hit the wires. Therefore, it is clear that the characteristic must have a maximum. The maximum is expected to be roughly at the grid voltage at which the diameter of the potential well is equal to the distance between two wires. Thus, the relevant parameter that determines the position of the maximum is expected to be the ratio between the Debye length and the distance between the wires. This is in qualitative agreement with the observations given in Table $I$.

Also the change in the grid characteristic in Fig. 4 with $V_{p}$ can be explained. With the plate electrically floating we expect and find a normal characteristic. When the plate is biased negatively, a sheath is formed in front of the plate. As long as this sheath does not come in contact with the sheath around the grid wires, no change is expected. At grid voltages 
just low enough so that the two sheaths touch each other, some of the ions trapped in the potential wells around the grid wires may hit the plate rather than the grid. Therefore, we expect to find a maximum in the characteristic around this point. This grid voltage depends on the plate voltage. A small negative value for $V_{p}$ requires a high negative $V_{0}$ value and vice versa. Therefore, the maximum of the characteristic moves to the right when $V_{p}$ is lowered. This is in agreement with the pictures in Fig. 4(a). At very negative plate voltages the potential well in front of the plate will reach the grid and no ions will be reflected. One would, therefore, expect the ion current to the grid to be nearly independent of $V$, as seen in the lower pictures in Fig. 4(a). This current is simply made up of the ions that hit the wires in their undisturbed paths through the grid.

The curve in Fig. 4(b) shows that the plate voltage $V_{p}^{\prime}$, for which the maximum of the grid characteristic disappears, is proportional to $n^{2 / 3}$. This fits our picture because the sheath thickness around a plate in a plasma is known to be determined by the spacecharge-limited current density given by $i \propto V^{3 / 2} / x^{2}$. In our case $x$ is the distance between grid and plate and $i=n\langle v\rangle$.

Finally, it is a consequence of our picture that it should be possible to form an echo with the reflected ions. This was demonstrated in Sec. IIID. The fact that the relative strengths of the transmitted and the reflected echoes are of the same order indicates that transmitted and reflected ions (at grid 2) suffer about the same spread in parallel velocity when passing $g_{2}{ }^{14}$

\section{INTERACTION BETWEEN REFLECTED PARTICLES AND PLASMA}

In this section we first describe experiments made in order to show that the reflected ions move as freely streaming particles. The experimental set-up was similar to that used in Sec. IIIB. The twin probe was placed at different distances from the pulsed grid, and pictures like the ones shown in Fig. 2(b) were taken. For densities less than $\sim 10^{11} \mathrm{~cm}^{-3}$ it was found that the width of the received signal was proportional to the distance between grid and probe, and that the amplitude was inversely proportional to this distance. Furthermore, the signals received at different distances were found to be similar. This is what would be expected if the signal were caused by freely streaming particles. A dis-

${ }^{14}$ T. H. Jensen, J. H. Malmberg, and T. M. O'Neil, Phys. Fluids 12, 1728 (1969).

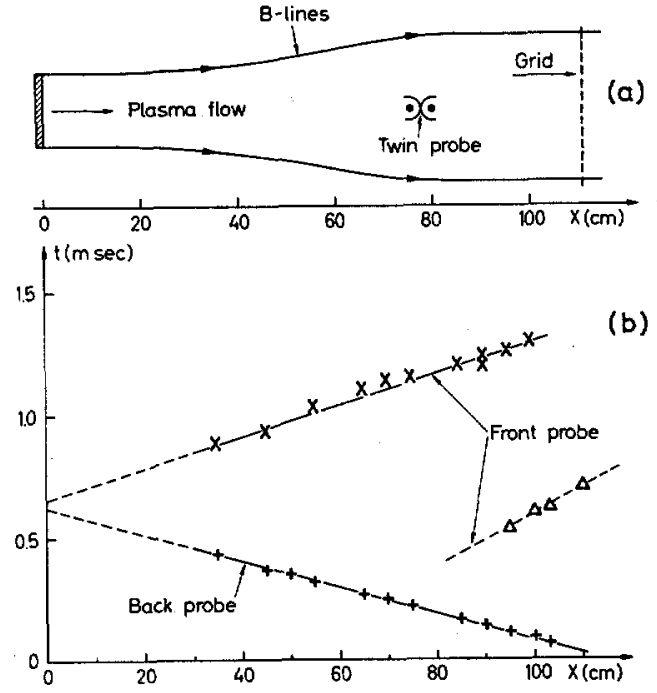

Fig. 5. (a) Schematic of set-up with magnetic mirror. (b) Time of arrival of pulses in this set-up. $n \approx 10^{10} \mathrm{~cm}^{-3}$.

cussion of the shape of a Landau-damped pulse as a function of the distance from the exciting grid will be given elsewhere. ${ }^{15}$

At higher densities, $n \gtrsim 10^{11} \mathrm{~cm}^{-3}$, the similarity between pulses received at different distances disappears. At long distances between grid and probe the signal is found to be smaller than expected, especially at that part which corresponds to lowvelocity particles. We assume that this is due to binary collisions, which, of course, are most effective for the slow particles. This suggests the possibility of using the technique described as a means of studying binary collisions in $Q$-machine plasmas.

In the low-density cases it was shown that the particles reflected from the grid can be reflected again either from the hot plate or from a magnetic mirror. Figure 5(a) shows the set-up used. The magnetic field was shaped like two regions of constant field. The grid was placed on the downstream side of the mirror. When pulsing the grid with a negative square pulse, we first see the normal reflected signal on the back probe. When placed between mirror and grid, the front probe thereafter receives two signals. When placed between mirror and hot plate, it only receives one signal. Figure 5 (b) shows the time of arrival of the pulse maximum as a function of position. We see that part of the pulse generated at the grid is reflected from the mirror and part from the hot plate.

Finally, a small sinusoidal voltage was applied to a negatively biased grid, and waves in the up-

15 P. Nielsen, Ris $\emptyset$ Report No. 190 (1969). 

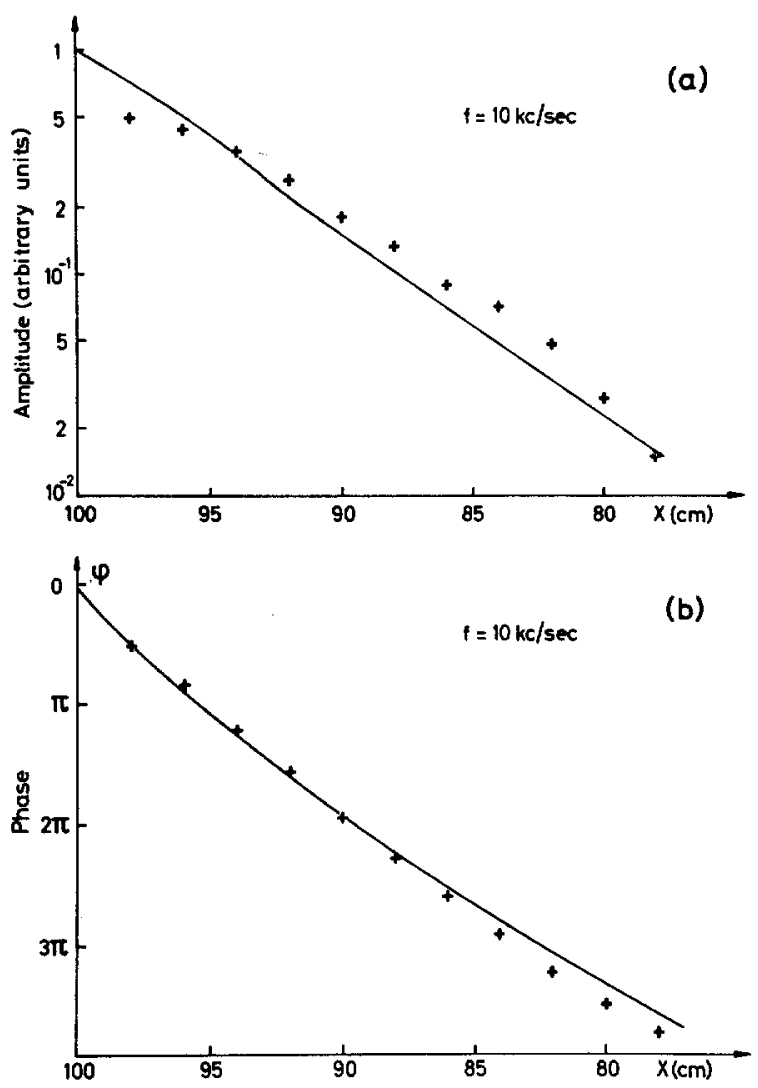

Fig. 6. + , Phase and amplitude of upstream wave. Solid curve calculated from a model with freely streaming particles. $n \approx 10^{10} \mathrm{~cm}^{-3}$. Grid position at $100 \mathrm{~cm}$.

stream direction were excited. The phase velocity and damping of these waves were studied by means of the back probe in a manner often used in the past. ${ }^{1-3}$ The signal from the back probe was analyzed by a PAR lock-in amplifier. When the grid bias was changed from floating to the voltage $V_{g \max }$ that corresponds to the maximum of the characteristic (see Sec. IIA), the phase velocity of the waves was found to increase from approximately $10^{5}$ to $2 \times 10^{5}$ $\mathrm{cm} / \mathrm{sec}$. The damping was found not to be strictly exponential, but, assuming exponential damping, our best estimate of the damping distance over wavelength, $\delta / \lambda$, was always at least 0.5 . For grid voltages lower than $V_{o \max }$ the phase velocity varied irregularly with $V_{g}$, but was normally higher than $2 \times 10^{5} \mathrm{~cm} / \mathrm{sec}$. Also the damping changed irregularly with $V_{g}$ in this region. Figure 6 shows the measured amplitude and phase as functions of the distance from the grid for a $10-\mathrm{kc} / \mathrm{sec}$ wave. For these measurements $V_{g}$ was around midway between floating and $V_{g \mathrm{max}}$. The calculations of the solid curves in Fig. 6 are based on a model where the wave is made up of freely streaming particles re- flected from the grid. The velocities of the reflected ions are assumed to follow a drifting Maxwellian distribution (compare Sec. IIIB). The curves were calculated on the assumption of a drift velocity equal to the ion thermal velocity $c_{i}\left[c_{i}=\left(2 \kappa T_{i} / m_{i}\right)^{1 / 2}=\right.$ $\left.0.56 \times 10^{5} \mathrm{~cm} / \mathrm{sec}\right]$.

Measurements such as the ones shown in Fig. 6 were taken for several frequencies and grid biases. For all measurements it was possible to find agreement with calculations based on the above-mentioned model. The drift velocities for which calculations gave the best fit to the experimental points were found to be in fair agreement with those measured in Sec. IIIB.

When the grid was operated at de voltages corresponding to curve $f$ in Fig. 2(b), the amplitude of a sinusoidal wave first increased when the probe was moved away from the grid, and then decreased. This corresponds to a bunching effect, which would be expected when a distribution function deduced from curve $f$ is used in a model of freely streaming particles.

\section{v. CONCLUSIONS}

The experiments described in this paper show that the interaction between a grid and a $Q$-machine plasma can be explained by a fairly simple physical picture. The reflection of ions is essentially caused by the potential barrier set up in the plasma around the grid. We have demonstrated that a pulse of reflected ions moves as freely streaming particles, which again can be reflected either from a hot plate or from a magnetic mirror. It should be noted that the interaction described here, being a dc interaction, differs from that giving rise to pseudowaves as reported by Yamashita et al. ${ }^{16}$ and by Longren et $a l .^{17}$

The wave experiments show that a grid can excite upstream waves with phase velocity and damping depending on the grid bias. The dispersion of these waves can be described in a model with freely streaming ions. To the best of our knowledge this kind of waves has not been observed in $Q$-machines before. In earlier experiments ${ }^{1-3}$ the phase velocity and the damping of the waves were found not to depend on details of the exciting mechanism; the waves could be explained as being natural modes in Landau's theory. It should be emphasized that our measurements need not be in contradiction of earlier measurements on upstream waves, especially those

${ }^{16}$ Y. Yamashita, H. Ikezi, N. Sato, and N. Takahashi, Phys. Rev. Letters 27 A, 79 (1968).

${ }_{17} \mathrm{~K}$. Longren, D. Montgomery, I. Alexeff, and W. D. Jones, Phys. Letters 25 A, 629 (1967). 
of Wong et al. ${ }^{1}$ We have operated our $Q$-machine with only one hot plate. The experiments of Wong et al. ${ }^{1}$ were performed in a $Q$-machine with two hot plates.

For a complete understanding of the interaction between grid and plasma to be gained, more work has to be done; especially, the plasma on the down- stream side of the grid should be examined. Work along these lines has been planned by our group.

\section{ACKNOWLEDGMENTS}

Thanks are due to H. K. Andersen and P. Michelsen for discussions during the work and to $M$. Nielsen and B. Reher for maintaining our $Q$-device. 